\title{
Update on modalities of mechanical ventilators
}

\section{A Greenough}

Recent advances in ventilator technology have often not been confirmed by randomised trials and instead serious shortcomings have been highlighted. Ventilation modes should only be introduced into routine clinical practice when proved efficacious in appropriately designed studies and no adverse outcomes identified by long term follow up. of infants is routinely treated with antenatal steroids and postnatal surfactant and have relatively compliant lungs. In such infants, whose respiratory efforts are further dampened by sedation, high frequency positive pressure ventilation may have fewer advantages, but this has not been tested. In infants fully ventilated after the first week, the results of physiological studies ${ }^{4}$ have shown that blood gas tensions are not improved by elevating ventilator rate above 60 breaths/min.

\section{PATIENT TRIGGERED VENTILATION (PTV)}

During triggered modes, the infant's spontaneous inspiratory effort can trigger positive pressure inflations. In synchronised intermittent positive pressure ventilation (SIPPV) or Assist/Control mode, any number of inflations can be triggered provided that the change in pressure, flow, or volume, etc exceeds the critical trigger level, whereas in synchronised intermittent mandatory ventilation (SIMV) only a preset number of inflations can be triggered regardless of the frequency of the infant's spontaneous respiratory efforts. In preterm infants with acute respiratory distress, physiological studies have shown that PTV compared with SIMV was associated with higher rates of synchrony, better blood gas tensions, higher tidal volumes, and lower work of breathing, fluctuations in blood pressure and cerebral blood flow velocity. These results suggested that use of PTV would reduce the incidence of both chronic lung disease (CLD) and intracerebral haemorrhage (ICH). Meta-analysis ${ }^{3}$ of the results of randomised trials surprisingly then showed that the only positive advantage of PTV was a shorter duration of ventilation. Possible explanations for the limited "success" of PTV include the fact that IPPV in the randomised trials had been successfully optimised to promote synchrony and avoid active expiration. Sedation is now administered to all ventilated infants and, by reducing respiratory efforts, may have influenced the results.

Finally, it is possible that suboptimal ventilators or triggering systems were employed. Differences in the performance of the currently tion) and shortening the inspiratory time tended to entrain the infant's respiratory efforts, such that inspiration and inflation coincided. This form of interaction was termed synchrony and was associated with improvement in blood gas tensions. ${ }^{2}$ Active expiration was reduced by increasing ventilator rate, which may explain the lower incidence of pneumothoraces in infants supported by high frequency positive pressure ventilation compared with IPPV in subsequent randomised trials. ${ }^{3}$ Active expiration is a manifestation of a respiratory reflex and is more common in infants with stiff lungs. The present population
Abbreviations: IPPV, intermittent positive pressure ventilation; PIP, peak inspiratory pressure; PEEP, peak end expiratory pressure; PTV, patient triggered ventilation; SIPPV, synchronised intermittent positive pressure ventilation; SIMV, synchronised intermittent mandatory ventilation; $C L D$, chronic lung disease; $\mathrm{ICH}$, intracerebral haemorrhage; PSV, pressure support ventilation; PAV proportional assist ventilation; VG, volume guarantee; VCV, volume cycled ventilation; HFOV, high frequency oscillation ventilation; CPAP, continuous positive airway pressure
Correspondence to: Professor Greenough Regional Neonatal Intensive Care, 4th floor, Ruskin Wing, King's College Hospital, London SE5 9RS, UK:

anne.greenough@kcl.ac.uk. 
available ventilators ${ }^{1}$ and triggering systems ${ }^{5}$ have been shown. Delivered volume is compromised in certain ventilators by increasing rate and reducing inflation time. ${ }^{1}$ In the trial that recruited the largest number of infants, ${ }^{6}$ no significant differences were seen overall, and, among very immature infants, a greater proportion of those supported by PTV compared with IPPV developed air leaks. Most of the infants in the trial $^{6}$ were supported by a ventilator incorporating an airway pressure trigger, which has been shown to have a significantly lower sensitivity and longer trigger delay than an airflow trigger when tested in very immature infants. ${ }^{5}$ Both triggering systems were used with the same ventilator and thus the differences noted were solely due to differences in the performance of the triggering systems. ${ }^{5}$ The relatively long trigger delay of the airway pressure trigger may explain the tendency to a higher air leak rate in the PTV group of the randomised trial. ${ }^{6}$ Currently, there is no evidence to suggest that PTV offers long term advantages over IPPV for preterm infants with acute respiratory distress syndrome, and it remains speculative whether improvements in ventilator and triggering system performance will alter outcome.

There have been no large randomised trials in which SIPPV and SIMV have been compared in acute respiratory distress, but SIMV appears to be more useful in infants with vigorous respiratory efforts as it avoids hypocarbia. In preterm infants recovering from respiratory distress syndrome, SIPPV is the more efficacious weaning mode. In a randomised trial, ${ }^{7}$ SIPPV resulted in a significantly shorter duration of weaning than IPPV. SIMV allows more flexible weaning than SIPPV because rate, pressure, or both can be reduced. Nevertheless, in three randomised studies comparing the efficacy of SIPPV with SIMV, ${ }^{89}$ although similar numbers of infants on each mode were successfully weaned, the duration of weaning was significantly longer on SIMV if the supported breath rate was reduced below 20 breaths/min. At low levels of ventilator support, oxygen consumption is increased ${ }^{10}$ : at least 20 breaths need to be supported to overcome the work of breathing imposed by the endotracheal tube.

Certain ventilators providing synchronised modes also allow monitoring of the delivered tidal volume. Thus, ventilatory support can be volume targeted by adjusting the PIP to maintain the delivered volume within a predetermined range. In a prospective randomised study, ${ }^{11}$ volume targeted SIPPV resulted in more consistent tidal volumes at lower respiratory rates than either intermittent mandatory ventilation or $\mathrm{SIMV}^{11}$; whether this translates into long term gains has not been tested.

\section{PRESSURE SUPPORT VENTILATION (PSV)}

During PSV, the patient triggers a pressure supported breath at a preset level. Certain neonatal ventilators used in this mode allow inflation to be terminated when the inspiratory flow is reduced to a certain level. For example, inflation is terminated when the flow is reduced to $15 \%$ of the maximum inspiratory flow when the Draeger Babylog 8000 (Draeger Medical, Luebeck, Germany) is used in PSV mode, and inflation can be terminated between $5 \%$ and $25 \%$ of maximum inspiratory flow if termination sensitivity is used with the Bird VIP (Bird Products, Palm Springs, California, USA). In a study of very immature infants, ${ }^{12}$ increasing the termination sensitivity to maximum almost eliminated asynchrony, but this was at the expense of reducing the inflation time. During the short study period, tidal volume was maintained, but this may have been at the expense of the infants increasing their respiratory efforts. Whether preterm infants are able to compensate for very short inflation times throughout their time on a ventilator was not tested, and randomised trials are required to determine whether this ventilation mode reduces air leaks. PSV outside the neonatal period has been shown to reduce the work of breathing during weaning, but it is important to be aware that, as with all modes, the effectiveness may be influenced by differences in ventilator performance. ${ }^{13}$

\section{PROPORTIONAL ASSIST VENTILATION (PAV)}

During PTV, ventilatory support is synchronised at the beginning and sometimes also at the end of inspiration; in contrast, during PAV the applied pressure is servocontrolled throughout each spontaneous breath. The applied pressure increases in proportion to the tidal volume and inspiratory flow generated by the patient and thus enhances the effects of the respiratory muscles on ventilation. ${ }^{14}$ The frequency, timing, and amplitude of lung inflation are controlled by the patient. ${ }^{14}$ The clinician can increase the "enhancement" to reduce the mechanical work of breathing; excessive enhancement, however, could result in overcompensation and overdistension. To prevent this happening, once the pressure preset inflation pressure is reached, the system aborts to PEEP. ${ }^{14}$ Problems can also occur if there is a large leak around the endotracheal tube, and there must be a "back-up" if the infant's respiratory efforts diminish. There are very few data on PAV in neonates. In one study, ${ }^{15}$ gas exchange during PAV was maintained with smaller transpulmonary pressures than during PTV or intermittent mandatory ventilation, but the infants were examined on each mode for only 45 minute periods.

\section{VOLUME GUARANTEE (VG)}

In VG mode, the PIP is servocontrolled so that the volume preset by the clinician is delivered during SIPPV, SIMV, or PSV. There is compensation for variations in spontaneous breathing, and increased patient effort results in less pressure applied by the ventilator, thus this mode may be particularly useful if the patient's respiratory compliance is changing rapidly. Adjustment is carried out from one breath to the next, the expiratory tidal volume is measured and compared with the desired volume, and a new pressure plateau is calculated for the next breath. If, however, the preset peak pressure is too low or there is no positive pressure plateau, either because the flow is too low or the inflation time is too short, the desired volume will not be delivered. Adequate ventilation can be achieved at lower airway pressures during VG, ${ }^{16}$ one explanation being the greater contribution by the infant's spontaneous respiratory efforts to minute ventilation. ${ }^{17}$ In addition, breath to breath variability may be lower in triggered ventilation modes with $\mathrm{VG}^{18}$ but this is not a universal finding. ${ }^{16}$ Theoretically, breath to breath adjustment could actually result in greater variability, for example if the infant had periodic breathing. Whether it would be better to base the adjustment on an average of breaths and how many breaths should be considered needs to be tested.

\section{VOLUME CYCLED VENTILATION (VCV)}

During VCV, regardless of changes in the infant's lung function, a constant volume is delivered. VCV and IPPV have been compared in randomised studies, although both were of small sample size. In one, ${ }^{19}$ VCV was associated with less hypotension and serious ICH, but IPPV was not delivered by comparable neonatal ventilators. In another, ${ }^{20}$ the duration of respiratory support was significantly shorter in infants supported by VCV, although the ventilator settings were manipulated so that volume delivery by the two modes was similar. The airway pressure waveforms used in both modes lacked a positive pressure plateau, ${ }^{21}$ which has been assumed to be important in facilitating gas exchange. VCV then needs to be tested against "standard" IPPV, with both modes being delivered by appropriate ventilators. ${ }^{19}$

\section{HIGH FREQUENCY OSCILLATION VENTILATION (HFOV)}

High frequency oscillators are characterised by having both active inspiratory and expiratory phases. Usually frequencies 
of $10-15 \mathrm{~Hz}$ are used, although reducing the frequency below $10 \mathrm{~Hz}$ can improve carbon dioxide elimination, because the delivered volume increases when rate is decreased. ${ }^{22}$ HFOV can be delivered alone or added to either or both of the inspiratory and expiratory phases of IPPV. In anecdotal series, gas exchange has been improved by using HFOV and IPPV together, but the combination will produce the greatest transpulmonary pressure swings and an important concern is that it may increase adverse outcomes related to volutrauma. Certain oscillators have a variable inspiratory to expiratory ratio, but increasing the inspiratory fraction from 30\% to $50 \%$ has not been shown in infants to increase gas trapping ${ }^{23}$ and it does elevate mean airway pressure and the delivered volume with consequent improvements in gas exchange. ${ }^{24}$

HFOV has been used with a low volume strategy, that is minimising pressures with the hope of preventing further trauma to the lungs and a high volume strategy during which a lung recruitment policy is employed. In homogeneous lung disease, the latter approach allows HFOV at low inspired oxygen concentrations, and evidence supports it as the preferred strategy. In surfactant deficient animals, "high volume" HFOV resulted in significantly less lung damage than either IPPV or low volume HFOV strategy. ${ }^{25}$ There have been no large randomised clinical studies comparing the two HFOV strategies, but a reduction in CLD has only been noted in trials in which the "high volume strategy" was used. There have been at least eight trials in which infants have been randomised to HFOV or "conventional" ventilation in the first 24 hours. They have differed with respect to antenatal steroid and postnatal surfactant usage, the exact timing of randomisation, and the type of conventional ventilation and ventilators employed. Importantly, no lung recruitment strategy was used in the control groups in most of the trials, and this may have influenced the outcomes, as pressure controlled ventilation with a sufficient level of PEEP and small driving pressure amplitudes is as effective as HFOV in maintaining optimal gas exchange, improving lung mechanics, and preventing protein influx. Meta-analysis of the results of the randomised trials ${ }^{26}$ has shown that HFOV, particularly if a lung recruitment strategy is used, is associated with a reduction in CLD. No significant differences in mortality were noted, but there was a trend towards an increase in air leak and ICH. ${ }^{26}$ There has only been one randomised trial comparing HFOV and IPPV in preterm infants with severe pulmonary dysfunction; a reduction in new pulmonary air leak, but no significant differences in the rate of pulmonary interstitial emphysema or of gross pulmonary air leak, mortality, or use of HFOV at 30 days was noted. The ICH rate, however, was increased in the infants supported by HFOV. ${ }^{27}$ There has also been only one randomised trial comparing HFOV and IPPV in infants with severe pulmonary dysfunction born at or near term, and no significant differences were seen between the two groups. $^{28}$

\section{"NASAL VENTILATION"}

It is beyond the scope of this review to discuss continuous positive airway pressure (CPAP). The evidence for the use of IPPV, SIMV, or HFOV delivered by nasal prongs will be considered, but, as the data are from anecdotal series or trials with only short term outcomes, it is not possible to conclude that these modes are useful. Randomised trials comparing nasal IPPV and nasal CPAP for apnoea of prematurity have yielded conflicting results. Meta-analysis ${ }^{29}$ showed no difference in carbon dioxide levels after four to six hours of support, and the systematic review highlighted that the trials did not report on gastrointestinal complications, although an association between the use of ventilation through nasal prongs and increased risk of gastrointestinal perforation had been previously noted. In a randomised trial, respiratory failure developing after extubation was considerably lower in infants supported by nasal SIMV rather than nasal CPAP, but only 41 infants were studied and a variety of definitions of respiratory failure were used. Nasal HFOV has been associated with reduction in carbon dioxide levels when applied to infants who developed moderate respiratory acidosis on nasal CPAP, but there were no randomised comparators and five of the 21 infants studied had to be intubated a few hours later because of carbon dioxide retention and a high oxygen requirement. ${ }^{31}$

\section{CONCLUSION}

Clinicians must be careful not to be seduced by "exciting" preliminary evidence. Results of physiological studies promised that both PTV and early HFOV would have advantages over "conventional" ventilation, but these were not confirmed by meta-analysis of subsequent randomised trials and instead serious shortcomings were highlighted. Ventilation modes should only be introduced into routine clinical practice when proved efficacious in appropriately designed studies and no adverse outcomes identified by long term follow up.

\section{REFERENCES}

1 Dimitriou G, Greenough A. Performance of neonatal ventilators. British Journal of Intensive Care 2002:in press.

2 Greenough A, Pool J, Greenall F, et al. Comparison of different rates of artificial ventilation in preterm neonates with the respiratory distress syndrome. Acta Paediatr Scand 1987;76:706-12.

3 Greenough A, Milner AD, Dimitriou G. Synchronized ventilation [Cochrane review]. In: The Cochrane Library, issue 1. Oxford: Update Software, 2001

4 Chan V, Greenough A, Hird MF. Comparison of different rates of artificial ventilation for preterm infants ventilated beyond the first week of life. Early Hum Dev 1991;26:177-83.

5 Dimitriou G, Greenough A, Cherian S. Comparison of airway pressure and airflow triggering systems using a single type of neonatal ventilator. Acta Paediatr 2001:90:445-7.

6 Baumer JH. International randomized controlled trial of patient triggered ventilation in neonatal respiratory distress syndrome. Arch Dis Child Fetal Neonatal Ed 2000;82:F5-10.

7 Chan V, Greenough A. Randomised controlled trial of weaning by patient triggered ventilation or conventional ventilation. Eur J Pediatr $1993 ; 152: 51-4$.

8 Chan V, Greenough A. Comparison of weaning by patient triggered ventilation or synchronous intermittent mandatory ventilation in preterm infants. Acta Paediatr 1994;83:335-7.

9 Dimitriou G, Greenough A, Giffin F, et al. Synchronous intermittent mandatory ventilation modes compared with patient triggered ventilation during weaning. Arch Dis Child Fetal Neonatal Ed 1995;72:F188-90.

10 Roze JC, Liet JM, Gournay V, et al. Oxygen cost of breathing and weaning process in newborn infants. Eur Respir J 1997;10:2583-5.

11 Mrozek JD, Bendel-Stenzel EM, Meyers PA, et al. Randomized controlled trial of volume-targeted synchronized ventilation and conventional intermittent mandatory ventilation following initial exogenous surfactant therapy. Pediatr Pulmonol 2000;29:1 1-18.

12 Dimitriou G, Greenough A, Laubscher B, et al. Comparison of airway pressure triggered and airflow triggered ventilation in very immature infants. Acta Paediatr 1998:87: 1256-60.

13 Sanders RC, Thurman TL, Holt SJ, et al. Work of breathing associated with pressure support ventilation in two different ventilators. Pediatr Pulmonol 2001;32:62-70.

14 Schulze A, Schaller P. Proportional assist ventilation: a new strategy for infant ventilation? Neonatal Respiratory Diseases 1996;6:1-10.

15 Schulze A, Gerhardt T, Musante G, et al. Proportional assist ventilation in low birth weight infants with acute respiratory disease: a comparison to assist/control and conventional mechanical ventilation. J Pediatr 1999; 135:339-44

16 Cheema IU, Ahluwalia JS. Feasibility of tidal volume-guided ventilation in newborn infants: a randomized, crossover trial using the volume guarantee modality. Pediatrics 2001;107:1323-8.

17 Herrera CM, Gerhardt T, Everett R, et al. Randomized, crossover study of volume guarantee (VG) versus synchronized intermittent mandatory ventilation (SIMV) in very low birth weight (VLBW) infants recovering from respiratory failure [abstract]. Pediatr Res 1994;45:304A.

18 Abubakar KM, Keszler M. Patient-ventilator interactions in new modes of patient-triggered ventilation. Pediatr Pulmonol 2001;32:71-5.

19 Piotrowski A, Sobala W, Kawczynski P. Patient-initiated, pressure-regulated, volume-controlled ventilation compared with intermittent mandatory ventilation in neonates: a prospective, randomised study. Intensive Care Med 1997;23:975-81.

20 Sinha SK, Donn SM, Gavey J, et al. Randomised trial of volume controlled versus time cycled, pressure limited ventilation in preterm infants with respiratory distress syndrome. Arch Dis Child Fetal Neonatal Ed 1997;77:F202-5. 
21 Greenough A, Milner A, Dimitriou G. Volume controlled and time cycled pressure limited ventilation. Arch Dis Child Fetal Neonatal Ed 1998;79:F79-80.

22 Laubscher B, Greenough A, Costeloe K. Performance of four neonatal high frequency oscillators. British Journal of Intensive Care 1996:6:148-52.

23 Alexander J, Milner AD. Determination of gas trapping during high frequency oscillatory ventilation. Acta Paediatr 1997;86:268-73.

24 Dimitriou G, Greenough A, Kavvadia V, et al. Comparison of two inspiratory: expiratory ratios during high frequency oscillation. Eur J Pediatr 1999:158:796-9.

25 McCulloch PR, Fokert PG, Froese AB. Lung volume maintenance prevents lung injury during high frequency oscillatory ventilation in surfactant deficient rabbits. Am Rev Respir Dis 1988;137: 1 185-92.

26 Henderson-Smart DJ, Bhuta T, Cools F, et al. Elective high frequency oscillatory ventilation versus conventional ventilation for acute pulmonary dysfunction in preterm infants [Cochrane review]. In: The Cochrane Library, issue 2. Oxford: Update Software, 2001
27 HIFO Study Group. Randomized study of high-frequency oscillatory ventilation in infants with severe respiratory distress. J Pediatr 1993; 122:609-19.

28 Clark RH, Yoder BA, Sell MS. Prospective, randomized comparison of high frequency oscillation and conventional ventilation in candidates for extracorporeal membrane oxygenation. J Pediatr 1994;124:447-54.

29 Lemyre B, Davis PG, De Paoli AG. Nasal intermittent positive pressure ventilation (NIPPV) versus nasal continuous positive airway pressure (NCPAP) for apnea of prematurity [Cochrane review]. In: The Cochrane Library, issue 2. Oxford: Update Software, 2001.

30 Friedlich $\mathbf{P}$, Lecart $C$, Posen $R$, et al. A randomized trial of nasopharyngeal synchronized intermittent mandatory ventilation versus nasopharyngeal continuous positive airway pressure in very low birth weight infants after extubation. J Perinatol 1999;19:413-18.

31 van der Hoeven $\mathbf{M}$, Brouwer $E$, Blanco $C E$. Nasal high frequency ventilation in neonates with moderate respiratory insufficiency. Arch Dis Child Fetal Neonatal Ed 1998;79:F61-3.

\section{Archimedes}

In order to give the best care to patients and families, paediatricians need to integrate the highest quality scientific evidence with clinical expertise and the opinions of the family. Archimedes is a bimonthly section in Archives which seeks to assist practising clinicians by providing "evidence based" answers to common questions which are not at the forefront of research but are at the core of practice.

The format of Archimedes may be familiar. A description of the clinical setting is followed by a structured clinical question and a brief report of the search. The best evidence available to answer the question is provided as a summary table (which is electronically linked to more detailed appraisals). To pull the information together, a commentary follows. Finally, to make it all much more accessible, a box provides the clinical bottom line.

This month the following topics have been published which may be of interest to neonatologists:

- Does iron have a place in the management of breath holding spells?

- Is omeprazole helpful in the management of children with reflux oesophagitis?

- Does oral sucrose reduce the pain of neonatal procedures?

Previous Archimedes questions can be found in the September 2001, November 2001, January 2002, and May 2002 issues of Archives. Readers wishing to submit their own questions - with best evidence answers-are encouraged to read the Instructions for Authors at http://www.archdischild.com. 